\title{
Electrochemical Sensors Integration for a non Invasive Breath Test for Early Diagnosis of Tropical Diseases
}

\author{
Dumitru Ulieru, Oana-Maria Ulieru,Xavi Vila, Alexandru Topor \\ 1 SITEX 45 SRL,114, GHICA TEI, BL.40,AP.2,DEPT.2, BUCHAREST,ROMANIA \\ e-mail address: ulierud@yahoo.com
}

\begin{abstract}
This topics aims at demonstrating the feasibility of electrochemical sensors application for a noninvasive, safe and patient-friendly methodology for on-site rapid diagnosis of tropical diseases. The proposed approach is based on breath samples analyses, which are easy to obtain and present no discomfort or risk for patients' health. In this study will be enrolled three different types of neglected tropical diseases from different geographical locations (Europe, South America and Nord Africa)
\end{abstract}

Key words: Detection, Chemical sensors, Nanomaterials, biomarkers, miniaturization,

\section{Generals}

Analytical chemistry methods will be employed for the identification of the breath volatile biomarkers of these diseases. Breath sampling will follow a standardised procedure A pool of potential nanomaterials with high affinity towards the identified volatile biomarkers will be selected (e.g.of gold nanoparticles, carbon nanotubes and semiconducting nanowires, functionalised with selected hydrophobic organic molecules, bio-molecules and/or metallic nanoparticles). For maximizing the possibility of success of our methodology, we will investigate the synergic effect of different types of Chemical Gas Sensors devices. These techniques are particularly attractive, since they can be miniaturised and are suitable for building on-site portable systems. Advanced pattern recognition algorithms will be employed for building discriminative models for the identification of the fingerprints of the different tropical diseases studied,

Tropical Diseases Detection with Chemical Sensing Devices.

For maximising the possibility of success of our methodology, we will investigate for the first time the synergic effect of different advanced and complementary nanomaterial-based techniques, all of them presenting the attractiveness that they can be miniaturised, thus being suitable for building on-site portable systems:

\section{Chemical Gas Sensors}

We employed different types of sensing transducers in order to investigate different (independent) nanoscale features of the nanomaterials exposed to the breath samples: changes in electrical/electronic properties (resistors, Schottky diodes, field effect transistors), mass (quartz crystal microbalances), noise (fluctuation-enhanced sensing), and dynamic modulation (temperature pulses). This way we will exploit a wide range of independent nanomaterials characteristics, which is expected to be highly beneficial for improving the overall sensitivity and selectivity of the CGS system and counteracting the interfering volatiles that could be present occasionally in (few) patients breath .

The selection of the most suitable CGS devices and sensing nanomaterials will be heuristically achieved by analysing successively CGS responses to the breath volatile biomarkers (i.e., pure chemical standards), vapour samples simulating the volatile patterns of the studied tropical diseases and real breath samples.

\section{Acknowledgments}

These works received funding from the European Union's Horizon 2020 research and innovation programme under the Marie Skłodowska-Curie grant agreement No 645758 project TROPSENSE". 